\title{
Efectos de un programa de danza española en mujeres con fibromialgia
}

\section{Effects of a spanish dance program on women with fibromyalgia \\ Efeitos de um programa de dança espanhola em mulheres com fibromialgia}

\author{
Chica, A., González-Guirval, F., Reigal, R.E., Carranque, G. y Hernández-Mendo, A. \\ Universidad de Málaga (España)
}

\begin{abstract}
RESUMEN
El objetivo de este estudio fue analizar los efectos de un programa de danza española sobre el nivel sintomático, la capacidad funcional, diversas variables psicológicas y la composición corporal en una muestra de mujeres con fibromialgia. Un total de 43 mujeres de 45 a 70 años participaron en el estudio. La intervención se realizó durante 6 semanas y 12 sesiones. Se utilizó un diseño intra-sujeto con medidas pre y post-test. Los instrumentos utilizados fueron la Escala Analógica Visual (VAS), el Cuestionario de Impacto de la Fibromialgia (FIQ), el Inventario de Depresión de Beck (BDI), el Inventario de Ansiedad Estado-Rasgo (STAI), la Escala Hamilton de Ansiedad (HARS), la Escala de Inteligencia Emocional (TMMS-24), el cuestionario Autoconcepto Forma 5 (AF5), el cuestionario de Perfil del Estado de Ánimo (POMS) y un bioimpediciómetro Tanita ${ }^{\circledR}$ BC-601. Los resultados mostraron efectos positivos después del programa, con mejoras en el estado de ánimo, la ansiedad psíquica y somática, así como la depresión. Por lo tanto, este tipo de intervenciones podrían contribuir a suavizar el impacto de la fibromialgia en las personas.
\end{abstract}

Palabras clave: Fibromialgia; danza; capacidad funcional; calidad de vida.

\section{ABSTRACT}

The objective of this study was to analyze the effects of a Spanish dance program on symptomatic level, functional capacity, psychological variables and body composition in a sample of women with fibromyalgia. A total of 43 women aged 45 to 70 years participated in the study. The intervention was performed for 6 weeks and 12 sessions. An intra-subject design with pre and post-test measurements was used. The instruments used were the Visual Analogue Scale (VAS), the Fibromyalgia Impact Questionnaire (FIQ), the Beck Depression Inventory (BDI), the State-Trait Anxiety Inventory (STAI), the Hamilton Anxiety Rating Scale (HARS), the Trait Meta-Mood Scale-24 (TMMS-24), the Self-concept form 5 questionnaire (AF5), the Profile of Mood States questionnaire (POMS) and a bioimpediciometer Tanita ${ }^{\circledR}$ BC-601. The results showed positive effects after the program, with improvements in mood, psychic and somatic anxiety, as well as depression. Therefore, these interventions could help to soften the impact of fibromyalgia in people.

Keywords: Fibromyalgia, dance, functional capacity, quality of life. 


\section{Danza española en mujeres con fibromialgia}

\section{RESUMO}

O objetivo deste estudo foi analisar os efeitos de um programa de dança espanhola no nível sintomático, capacidade funcional, diversas variáveis psicológicas e composição corporal em uma amostra de mulheres com fibromialgia. Um total de 43 mulheres com idade entre 45 e 70 anos participaram do estudo. A intervenção foi realizada por 6 semanas e 12 sessões. Um desenho intra-sujeito foi usado com medições pré e pós-teste. Os instrumentos foram a Escala Analógica Visual (VAS), o Impact Questionnaire Fibromialgia (FIQ), o Beck Depression Inventory (BDI) Ansiedade Traço Estado Inventory (IDATE), Hamilton Anxiety Scale ( HARS), Escala de inteligência emocional (TMMS-24), o Autoconcepto Form 5 (AF5) questionário Mood States (POMS) Perfil questionário e bioimpediciómetro Tanita ${ }^{\circledR}$ BC-601. Os resultados mostraram efeitos positivos após o programa, com melhorias no humor, ansiedade e depressão psíquica e somática. Portanto, esse tipo de intervenção poderia ajudar a amenizar o impacto da fibromialgia nas pessoas.

Palavras chave: Fibromialgia; dança; capacidade funcional; qualidade de vida.

\section{INTRODUCCIÓN}

La fibromialgia (FM) se trata de una enfermedad de etiología desconocida, caracterizada por la presencia de dolor crónico generalizado en zonas musculares, tendinosas, articulares y viscerales (Restrepo y Rojas, 2011). Fue reconocida como enfermedad desde 1992 por la Organización Mundial de la Salud (OMS), incluyéndose en el manual de Clasificación Internacional de Enfermedades (CIE 10) bajo el código M79.04 (World Health Organization, 2010). Se ha calculado que aproximadamente un $2-5 \%$ de la población mundial padece FM (Busse et al., 2013). En España existen casi 2 millones de afectados, con un diagnóstico de 120.000 casos por año (Escudero et al., 2010), lo que supone entre el 2 y el $2,7 \%$ de la población mayor de 20 años, con una mayor incidencia en mujeres $(4,2 \%)$ que en hombres $(0,2 \%)$.

Las personas que padecen FM manifiestan alteraciones a nivel biológico, psicológico y social, que inciden negativamente en su calidad de vida (Palstam, Gard y Mannerkorpi, 2013). Pueden presentan síntomas como fatiga, depresión, ansiedad, rigidez articular, cefalea, sensación de tumefacción en manos, trastornos del sueño, parestesias en extremidades, trastornos de pánico e hipocondría (Goldenberg, 1999; Humphrey et al., 2010). Asimismo, podría conllevar hipersensibilidad en los denominados puntos gatillo o tender points, correspondientes a zonas fisiológicamente más sensibles a la presión, aunque podrían no presentar daño en músculos, ligamentos, tendones o articulaciones, dificultando su diagnóstico (Rodríguez, Orta y Amashta, 2010).
Cuando se interviene con estas personas han de utilizarse protocolos que valoren su naturaleza crónica, la incertidumbre de su evolución o su incidencia social (Rodham, Rance y Blanke, 2010). A lo largo de los años se han empleado procedimientos farmacológicos y no farmacológicos, entre los que se encuentran los basados en el ejercicio físico, los cuales han puesto de manifiesto beneficios como mejoras en el estado del aparato locomotor, en el funcionamiento del sistema cardiovascular, favorecimiento del sueño, mejora de la sensación de fatiga, mejor autoconcepto, así como disminución del dolor, la ansiedad o los síntomas depresivos (Estévez-López et al., 2015; Soriano-Maldonado, 2015).

Cuando se utilizan programas de ejercicio físico en FM, habría que tener en cuenta posibles efectos adversos como dolor, fatiga, rigidez o riesgo de caídas (Aparicio et al., 2015; Goes et al., 2012), así como aquellos que permitan alcanzar los objetivos de tratamiento (Busch, Schachter, Overend, Peloso y Barber, 2008). Entre los programas específicos que han mostrado beneficios en FM se pueden señalar los siguientes: mejoras en la capacidad funcional, disminución del dolor y mejora de la calidad de vida combinando ejercicios en seco y en agua, (Santos, 2013); mejoras en el estado de ánimo con entrenamiento aeróbico (Assis et al., 2006); descenso de depresión y ansiedad, con trabajo aeróbico y trabajo de fuerza (Tomas-Carús et al., 2008); mejoras en capacidad funcional con el uso del método pilates (Altan, Korkmaz, Bingol y Gunay, 2009).

En este contexto pueden recogerse diferentes propuestas de ejercicio, aunque Carbonell-Baeza, Aparicio, Álvarez-Gallardo y Delgado-Fernández 


\section{Chica, A., González-Guirval, F., Reigal, R.E., Carranque, G. y Hernández-Mendo, A.}

(2014), con el objetivo de sentar las bases claves para la prescripción de programas de actividad física en pacientes con FM, tanto realizados en agua como en seco, publica el "Plan para la Promoción de la Actividad Física y la Alimentación Equilibrada (PAFAE). Tras analizar diferentes estudios y valorar una serie de pautas necesarias para implementar ejercicio físico en FM, consideran que hay que tener también en cuenta el concepto de individualización en cuanto al tipo de esfuerzo, la intensidad y la duración (de cada sesión), así como la densidad (relación Trabajo/Descanso o recuperación), la frecuencia del ejercicio y la capacidad física de cada paciente.

En las últimas décadas la danza ha pasado de ser utilizada en ambientes escolares y artísticos, a formar parte también de centros asistenciales y de rehabilitación, empleada en salud mental, medicina y programas de promoción de la salud, (e.g. Reca, 2005). En adultos de edad avanzada, es capaz de mejorar la potencia aeróbica, resistencia muscular, fuerza, flexibilidad del tren inferior, equilibrio y agilidad tanto estáticos como dinámicos y velocidad de la caminada (Keogh, Kilding, Pidgeon, Ashley y Gillis, 2009). También se ha encontrado que, tras un programa de danza aeróbica, desciende el tejido graso subcutáneo y la composición corporal en mujeres jóvenes, (Pantelic, Milanovic, Sporis y StojanovicTosic, 2013). Asimismo, a nivel psicológico y social se han observado asociaciones positivas como la disminución de la depresión y el insomnio, la mejora en la liberación de emociones y en la creatividad, y el aumento de la felicidad y la alegría (Alpert, 2011; Knestaut, Devine y Verlezza, 2010; Pinniger, Thorsteinsson, Brown y McKinley, 2013).

Debido a los efectos positivos de la danza en otras poblaciones, se considera que podrían tener beneficios positivos en pacientes de FM. Por ello, el objetivo de este estudio fue analizar los efectos de un programa de danza española sobre el nivel sintomático, la capacidad funcional, diversas variables psicológicas y la composición corporal en una muestra de mujeres con fibromialgia. Se determinará la existencia de mejoras significativas en las variables analizadas tras la aplicación del programa de danza española propuesto.

\section{MATERIAL Y MÉTODOS}

Participantes
Los participantes del estudio fueron 43 mujeres con fibromialgia (Asociación Afibroma, Málaga, $n=13$; Asociación Afinsyfacro, Madrid, $n=25$; Asociación Fibrocampiña, Marchena, $n=5$ ), con edades comprendidas entre los 45 y los 70 años, $(M=32.95$; $D T=6.59)$. Se eliminaron 10 participantes por no cumplir alguno de los siguiente criterios: no cumplimentar adecuadamente los cuestionarios o por imposibilidad de realizar actividad física por motivos de salud. Se obtuvo previamente informe positivo del comité ético de experimentación de la Universidad de Málaga, ( $\mathrm{N}^{\circ} 193, \mathrm{~N}^{\circ}$ de Registro en CEUMA: 2014007-H), y el consentimiento informado. Además, durante todo el proceso de investigación se respetaron los principios éticos de la Declaración de Helsinki (World Medical Association, 2013), así como los recogidos en el Manual de la Fibromialgia de la Consejería de Salud (Carballo et al., 2005), en su apartado del ejercicio físico.

\section{Instrumentos}

a) Composición corporal. Para analizar esta variable se utilizó un bioimpedianciómetro Tanita BC-601. Con el objetivo de minimizar el margen de error de los resultados obtenidos con la Báscula Tanita BC-601, se tuvieron en cuenta una serie de pautas $y$ recomendaciones propuestas por George, Fisher, y Vehrs (2007): no realizar ejercicios agotadores el día anterior, no alterar significativamente la alimentación el día previo a la prueba, vestir ropa cómoda y controlar la toma de medicamentos que puedan alterar los niveles de agua corporal. El dato de la altura necesaria para analizar la composición corporal se evaluó con un tallímetro convencional.

b) El nivel sintomático, se evaluó a través de la Escala Visual Analógica (EVA) adaptada al español por Lázaro, Bosch, Torrubia y Baños (1994). Se compone de cuatro escalas analógicas visuales, correspondientes a las dimensiones de la calidad de vida (física, psicológica y social), que conforman el núcleo principal de la afectación en la FM. Las respuestas, que pueden puntuar de 1 a 10 se transforman posteriormente en un valor total.

c) Se midió la capacidad funcional, en relación al grado de incapacitación o disfunción que los síntomas del dolor fibromialgico produjeron en la semana previa a la toma de datos, se evaluó mediante el Cuestionario de Impacto de la Fibromialgia (FIQ, 


\section{Danza española en mujeres con fibromialgia}

Burckhardt, Clark y Bennett, 1991) validada al español (Monterde, Salvat, Montull, y Fernández, 2004). Se compone de 10 ítems que evalúan diferentes aspectos, relativos a la semana previa a su realización, como la capacidad funcional, lo bien que se sienten, los días de absentismo laboral, percepción del dolor, fatiga, alteraciones del sueño, rigidez, tensión o tristeza. Los ítems pueden valorarse desde 0 (ningún impacto de la enfermedad) a 10 (máximo impacto de la enfermedad). Para obtener el índice global del impacto o afectación, se suman las puntuaciones transformadas de las diez escalas.

d) La inteligencia emocional se evaluó a través de la Escala de Inteligencia Emocional (TMMS, Salovey, Mayer, Goldman, Turvey y Palfai, 1995) en su versión validada al español de 24 items, (TMMS-24, Fernandez-Berrocal, Extremera y Ramos, 2004). La escala original es una escala rasgo que evalúa el metaconocimiento de los estados emocionales mediante 48 items, concretamente, las destrezas con las que podemos ser conscientes de nuestras emociones y de nuestra capacidad para regularlas. A este cuestionario se responde mediante escala tipo Likert de 1 (nada de acuerdo) a 5 (totalmente de acuerdo). La TMMS-24 contiene tres dimensiones claves de la IE con 8 items cada una de ellas: Percepción emocional, comprensión de sentimientos y regulación emocional. Los análisis de consistencia interna fueron adecuados (Alfa de Cronbach), con valores entre .85 y .90 .

e) La ansiedad estado y rasgo se evaluaron a través del Cuestionario de Ansiedad Estado-Rasgo (STAI, Speilberger, 1983). Consta de 40 ítems y dos factores: ansiedad estado (AE) y ansiedad rasgo (AR). Las puntuaciones en cada uno pueden variar desde 0 a 60 puntos. Se contesta cada item en base a 4 niveles 0,1 , 2 y 3 , relativos a la ansiedad estado o la ansiedad rasgo. Los análisis de consistencia interna fueron adecuados (Alfa de Cronbach) (ansiedad estado, $\alpha=.80$; ansiedad rasgo, $\alpha=.75$ ).

f) El autoconcepto se evaluó a través del Cuestionario Autoconcepto Forma-5 (AF5, García y Musitu, 2001), basado en el modelo teórico de Shavelson, Hubner y Stanton (1976). Compuesto por 30 elementos, evalúa las dimensiones académico/laboral, social, familiar y físico. Los participantes pueden responder cada ítem con una valor entre 1 (totalmente en desacuerdo) y 99 (totalmente de acuerdo). Los análisis de consistencia interna fueron adecuados (Alfa de Cronbach), con valores entre .75 y .87 .

g) El estado de ánimo se evaluó a través del cuestionario de Perfil de Estados de Ánimo, (POMS, McNair, Lorr y Dorppleman, 1971), en su traducción al castellano (Arce, Adrade y Seoane, 2000). Se trata de una lista compuesta por 65 adjetivos, diseñada inicialmente para el ámbito clínico, se extendió posteriormente a otras áreas. Este instrumento evalúa las dimensiones tensión, depresión, cólera, vigor, fatiga, confusión y amistad. Las respuestas se contestan con una escala entre 0 (nada) y 4 (muchísimo). Los análisis de consistencia interna fueron adecuados (Alfa de Cronbach), con valores entre .74 y .92 .

h) La ansiedad psíquica y la ansiedad somática, se evaluaron a través de la Escala de ansiedad de Hamilton (HARS, Hamilton, 1959) validada al español, (Lobo et al, 2002). La escala cuenta con 14 ítems y dos factores, ansiedad psíquica y ansiedad somática. A cada ítem se contesta con una puntuación desde 0 (ausencia del síntoma) y 4 (síntoma muy grave o incapacitante). La puntuación total del instrumento puede oscilar desde 0 (ausencia de ansiedad) a 56 (máximo grado de ansiedad). Los análisis de consistencia interna fueron adecuados (Alfa de Cronbach), (ansiedad psíquica, $\alpha=.85$; ansiedad somática, $\alpha=.86$ ).

i) La depresión se evaluó a través del Inventario de Depresión de Beck (BDI; Beck, Ward, Mendelson, Monck y Erbaugh, 1961). Consta de 21 ítems que miden aspectos cognitivos, afectivos y factores neurovegetativos que tienen que ver con la depresión, proporcionando cada pregunta cuatro opciones de respuesta con una puntuación entre 0 y 3 , que representan el estado en el que se ha encontrado en la última semana. Los análisis de consistencia interna fueron adecuados (Alfa de Cronbach), con un valor de .87 .

\section{Procedimiento}

Se empleó un diseño de tipo intra-sujeto, con medidas pre-post. Para la selección de la muestra se contactó con diferentes asociaciones Afibroma (Málaga), Afinsifacro (Madrid) y Fibrocampiña (Marchena). Se informó mediante correo electrónico del estudio que se iba a realizar y se citó a las personas interesadas 


\section{Chica, A., González-Guirval, F., Reigal, R.E., Carranque, G. y Hernández-Mendo, A.}

para explicarles el procedimiento que se llevaría a cabo.

Se efectuaron dos evaluaciones, en las cuales se analizaron parámetros de condición física y se rellenaron los cuestionarios, que eran autoadministrables. Los investigadores estuvieron presentes para resolver posibles dudas.

En la fase de intervención, los grupos no superaron los 15 participantes para un mayor control y feedback sobre la tarea realizada. La duración fue de 12 sesiones de 60 minutos en un periodo de 6 semanas, estando el programa de actividad basado en coreografías de danza española. La estructura de cada sesión fue: 15 minutos para el calentamiento, 25 minutos para el aprendizaje de nuevas dinámicas y montaje de la coreografía, 15 minutos de vuelta a la calma y 5 minutos dedicados a recibir feedback de las sensaciones experimentadas durante la clase. Además, la actividad física tuvo una intensidad baja, para un menor impacto en los participantes dadas sus características. En ellas los ejercicios implicaban fundamentalmente cualidades como la flexibilidad, la fuerza-resistencia, el equilibrio y la resistencia aeróbica, así como un adecuado control cognitivo para la gestión de las coreografías aprendidas.

\section{Análisis estadístico}

Los datos fueron sometidos a análisis descriptivos e inferenciales. Se comprobó, la normalidad de los mismos (Shapiro-Wilk) y el índice de consistencia interna de las diferentes escalas (Alfa de Cronbach). Para analizar las diferencias entre grupos se utilizaron las técnicas de t-student y Wilcoxon. Para el procesamiento estadístico de los datos se ha usado el programa informatizado SPSS en su versión 20.0.

\section{RESULTADOS}

\section{Variables físicas}

En la tabla 1 se muestran los valores de asimetría, curtosis y prueba de Shapiro-Wilk.

Tabla 1. Valores de asimetría, curtosis y Shapiro-Wilk para las medidas de condición física

\begin{tabular}{|c|c|c|c|c|c|c|}
\hline & \multicolumn{3}{|c|}{ Pre-test } & \multicolumn{3}{|c|}{ Post-test } \\
\hline & $A$ & $K$ & $\begin{array}{l}S- \\
W\end{array}$ & $A$ & $K$ & $S-W$ \\
\hline Peso & .58 & .35 & .94 & .58 & .35 & .94 \\
\hline IMC & .57 & .17 & .94 & .57 & .17 & .94 \\
\hline$\% \mathrm{GC}$ & -.89 & .95 & .96 & -.89 & .95 & .97 \\
\hline$\% \mathrm{GV}$ & .03 & -.50 & .97 & .03 & -.50 & .93 \\
\hline MM & .30 & -.08 & .99 & .30 & -.08 & .93 \\
\hline EM & -.16 & -1.11 & .95 & -.16 & -1.11 & .95 \\
\hline
\end{tabular}

Nota. IMC = Índice de Masa Corporal; GC = Grasa Corporal; $\mathrm{GV}=$ Grasa Visceral; $\mathrm{MM}=$ Masa Muscular;

$\mathrm{EM}=$ Edad Metabólica.

En la tabla 2 se muestran las medias y desviaciones típicas de los factores analizados, así como las diferencias antes y después de la intervención. Los análisis efectuados indicaron que no hubo diferencias en ninguna variables $(p>.05)$.

Tabla 2. Medias, desviaciones típicas y comparación de medias para las medidas de condición física

\begin{tabular}{|c|c|c|c|c|c|}
\hline & \multicolumn{2}{|c|}{ Pre-test } & \multicolumn{2}{|c|}{ Post-test } & \multirow[b]{2}{*}{ Sig. } \\
\hline & $M$ & $D T$ & $M$ & $\overline{D T}$ & \\
\hline Peso & 66.78 & 11.87 & 66.62 & 11.8 & n.s. \\
\hline IMC & 27.90 & 4.87 & 27.62 & 4.78 & n.s. \\
\hline$\% \mathrm{GC}$ & 36.01 & 8.04 & 36.76 & 5.64 & n.s. \\
\hline$\% \mathrm{GV}$ & 8.59 & 3.32 & 8.37 & 2.78 & n.s. \\
\hline $\mathrm{MM}$ & 39.54 & 4.83 & 39.32 & 4.80 & n.s. \\
\hline EM & 55.59 & 14.96 & 54.93 & 13.4 & n.s. \\
\hline
\end{tabular}

\section{Variables psicológicas}

En la tabla 3 se pueden observar los valores de asimetría, curtosis y prueba de Shapiro-Wilk. Hubo problemas de normalidad para autoconcepto social, autoconcepto familiar y ansiedad psíquica. 


\section{Danza española en mujeres con fibromialgia}

Tabla 3. Valores de asimetría, curtosis y prueba de Shapiro-Wilk para las medidas de evaluación sintomáticas, capacidad funcional y variables psicológicas

\begin{tabular}{|c|c|c|c|c|c|c|}
\hline & \multicolumn{3}{|c|}{ Pre-test } & \multicolumn{3}{|c|}{ Post-test } \\
\hline & $A$ & $K$ & $S-W$ & $A$ & $K$ & $S-W$ \\
\hline EVA & -1.08 & .22 & $.69 * * *$ & -.47 & -.73 & $.78 * * *$ \\
\hline FIQ & -.09 & -.38 & .96 & -.07 & -.96 & .95 \\
\hline \multicolumn{7}{|l|}{ TMMS } \\
\hline Percepción & -.10 & -.12 & .98 & .11 & -.84 & .97 \\
\hline Comprensión & .15 & -.85 & .96 & -.09 & -.64 & .96 \\
\hline Regulación & -.01 & -1.27 & .94 & -.12 & -.77 & .96 \\
\hline \multicolumn{7}{|l|}{ STAI } \\
\hline Ansiedad-estado & -.49 & .20 & .95 & -.31 & 1.40 & .96 \\
\hline Ansiedad-rasgo & -.55 & 2.01 & .93 & -.10 & 1.54 & .93 \\
\hline \multicolumn{7}{|l|}{ AF5 } \\
\hline Académico-laboral & -.49 & .18 & .95 & -.78 & .06 & .94 \\
\hline Social & .04 & -.42 & .98 & .29 & -.61 & $.92 *$ \\
\hline Emocional & .62 & -.33 & .95 & .24 & -.20 & .97 \\
\hline Familiar & -.16 & -1.04 & .96 & -.60 & -1.02 & $.90^{*}$ \\
\hline Física & .05 & -.86 & .97 & .13 & -.68 & .97 \\
\hline \multicolumn{7}{|l|}{ POMS } \\
\hline Tensión-ansiedad & .12 & -.41 & .97 & .34 & -.83 & .95 \\
\hline Depresión & .05 & -.98 & .95 & .62 & -.28 & .95 \\
\hline Angustia & .05 & -.75 & .97 & .82 & .17 & .93 \\
\hline Vigor & .53 & .72 & .96 & .60 & -.18 & .96 \\
\hline Fatiga & -.05 & .16 & .98 & -.20 & -1.11 & .96 \\
\hline Confusión & .25 & -.40 & .97 & .46 & -.70 & .93 \\
\hline \multicolumn{7}{|l|}{ HARS } \\
\hline Ansiedad-psíquica & -.64 & -.43 & $.90^{*}$ & -.50 & .08 & .96 \\
\hline Ansiedad-social & .20 & -1.15 & .94 & .24 & -.63 & .96 \\
\hline Total & -.22 & -.58 & .95 & .13 & -.49 & .97 \\
\hline BDI & .17 & -.17 & .98 & -.17 & -1.18 & .94 \\
\hline
\end{tabular}

Nota. $\mathrm{A}=$ Asimetría; $\mathrm{K}=$ Curtosis; $\mathrm{S}-\mathrm{W}=$ Shapiro-Wilk.

$* p<.05 ; * * * p<.001$

En la tabla 4 se muestran las medias y desviaciones típicas de cada uno de los factores analizados, así como las diferencias entre las evaluaciones. Como se pueden apreciar, hubo diferencias significativas en las medidas del POMS tensión-ansiedad $(t=3.52 ; p$ $<.01)$, depresión $(t=5.14 ; \mathrm{p}<.001)$, angustia $(t=$ 


\section{Chica, A., González-Guirval, F., Reigal, R.E., Carranque, G. y Hernández-Mendo, A.}

$3.01 ; p<.01)$, fatiga $(t=2.63 ; p<.05)$ y confusión $(t$ $=4.52 ; p<.001)$, del BDI $(t=3.66 ; p<.01)$, así como del HARS ansiedad psíquica $(Z=-4.16 ; p<.001)$, ansiedad somática $(t=5.08 ; p<.001)$ y total $(t=4.90$; $p<.01)$. Asimismo, hubo valores próximos a la significación en EVA $(Z=-1.80 ; p=.073)$, FIQ $(t=$ $2.01 ; p=.054)$ y del AF5 la dimensión física $(t=$ $1.92 ; p=.063)$.

Tabla 4. Medias, desviación típica, comparación de medias y tamaño del efecto para las medidas de evaluación sintomática, capacidad funcional y variables psicológicas

\begin{tabular}{|c|c|c|c|c|c|}
\hline & \multicolumn{2}{|c|}{ Pre-test } & \multicolumn{2}{|c|}{ Post-test } & \multirow[b]{2}{*}{ Sig. } \\
\hline & $M$ & $D T$ & $M$ & $D T$ & \\
\hline EVA & 2.55 & .62 & 2.30 & .68 & al \\
\hline FIQ & 6.60 & 1.46 & 5.98 & 1.62 & b1 \\
\hline \multicolumn{6}{|l|}{ TMMS } \\
\hline Percepción & 22.21 & 7.90 & 21.18 & 6.28 & n.s. \\
\hline comprensión & 25.55 & 7.70 & 24.21 & 6.69 & n.s. \\
\hline Regulación & 26.67 & 7.97 & 27.67 & 7.09 & n.s. \\
\hline \multicolumn{6}{|l|}{ STAI } \\
\hline Ansiedad-estado & 14.94 & 3.19 & 15.24 & 3.08 & n.s. \\
\hline Ansiedad-rasgo & 16.52 & 3.10 & 15.58 & 2.49 & n.s. \\
\hline \multicolumn{6}{|l|}{ AF5 } \\
\hline Académico-laboral & 6.03 & 2.34 & 11.19 & 19.47 & n.s. \\
\hline Social & 5.62 & .89 & 5.67 & .92 & n.s. \\
\hline Emocional & 4.12 & 2.19 & 4.56 & 2.20 & n.s. \\
\hline Familiar & 4.95 & 1.51 & 5.14 & 1.48 & n.s. \\
\hline Física & 4.43 & 2.14 & 4.93 & 1.97 & $\mathrm{cl}$ \\
\hline \multicolumn{6}{|l|}{ POMS } \\
\hline Tensión-ansiedad & 53.03 & 9.59 & 48.70 & 10.44 & $* *^{2}$ \\
\hline Depresión & 59.59 & 11.03 & 52.03 & 9.11 & $* * *^{2}$ \\
\hline Angustia & 61.22 & 11.48 & 55.91 & 10.17 & $* *^{2}$ \\
\hline Vigor & 51.59 & 8.89 & 51.30 & 8.36 & n.s. \\
\hline Fatiga & 58.34 & 9.06 & 54.09 & 10.10 & $* 1$ \\
\hline Confusión & 48.16 & 10.59 & 43.03 & 8.95 & $* * *^{2}$ \\
\hline \multicolumn{6}{|l|}{ HARS } \\
\hline Ansiedad-psíquica & 15.30 & 5.51 & 11.27 & 4.80 & $* * *^{2}$ \\
\hline Ansiedad-social & 15.82 & 5.93 & 11.73 & 5.81 & $* * *^{2}$ \\
\hline Total & 30.30 & 9.77 & 23.18 & 9.51 & $* * *^{2}$ \\
\hline BDI & 20.55 & 9.58 & 16.84 & 8.29 & $* * *^{2}$ \\
\hline
\end{tabular}

Nota. n.s. $=$ no significativo.

${ }^{\mathrm{a}}=.073 ;{ }^{\mathrm{b}}=.054 ;{ }^{\mathrm{c}}=.063 ;{ }^{*} p<.05 ; * * p<.01 ; * * * p<.001$

$d^{\prime}$ Cohen: ${ }^{1} d>.20 ;{ }^{2} d>.50 ;{ }^{3} d>.80$

\section{DISCUSIÓN Y CONCLUSIONES}




\section{Danza española en mujeres con fibromialgia}

El objetivo del presente estudio fue analizar los efectos de un programa normalizado de danza española sobre el nivel sintomático, la capacidad funcional, diversas variables psicológicas y la composición corporal en una muestra de mujeres con fibromialgia (FM). Los resultados hallados en este trabajo indican que el programa de danza española produjo mejoras en el estado de ánimo, la depresión y el nivel de ansiedad psíquico y somático. Estos datos contribuyen a considerar que la práctica de actividad física, en concreto la danza, podrían ayudar a las personas que padecen fibromialgia a mejorar su calidad de vida.

Los resultados encontrados en estado de ánimo se muestran congruentes con otros estudios que aplicaron programas de intensidad moderada basados en entrenamiento aeróbico (Assis et al., 2006) o fuerza (Häkkinen, Häkkinen, Hannonen y Alen, 2001). Asimismo, la danza es propensa a realizarse en un contexto de diversión y recreación (Gardner, 1991), lo cual sería propicio para influir positivamente en el estado de ánimo (Bernardes, 2011). En esta línea, Autores como Peluso y Andrade (2005) consideran que actividades con características complacientes son más proclives a las mejoras del estado de ánimo que las estrictas y rígidas. Por su parte, los resultados en el parámetro depresión se encuentran en línea con otros trabajos con población con fibromialgia (LópezRodríguez, Castro-Sánchez, Fernández-Martínez, Matarán-Peñarrocha y Rodríguez-Ferrer, 2012; Tomás-Carús et al., 2008).

Los resultados hallados en el Cuestionario de Impacto de la Fibromialgia están en la línea con otros estudios (Kingsley et al., 2005; Nørregaard, Lykkegaard, Mehlsen y Danneskiold-Samsøe, 1997), que no consiguieron resultados significativos tras un programa de baile y otras actividades físicas. Sin embargo, se muestran contrarios a otros que si reflejaron resultados positivos (Carbonell, 2010; Frade, 2011; Mungia, Alegre, Serverto y Legaz, 2007; Pinzón, Angarita y Correa, 2013; Romero, 2012; Santos, 2013). Aunque existe bastante variabilidad en los resultados encontrados, podría sugerirse que la mayor parte de los programas de actividad física que obtienen resultados positivos implementan un programa de mayor duración. Por ello, se propone intervenciones más prolongadas para seguir profundizando en los efectos de la danza sobre estas variables.
Los resultados en ansiedad reflejan mejoras significativas en ansiedad psíquica y somática, lo cual sería congruente con otros con pacientes de fibromialgia (Blázquez, 2015; Sencan et al., 2004). Sin embargo, no se muestran diferencias en ansiedad estado y rasgo, lo cual podría deberse a una mayor sensibilidad del test de Hamilton para evaluar los cambios producidos tras un tratamiento y la evolución del trastorno a lo largo del tiempo (Lobo et al., 2002). Por otro lado, el nivel sintomático evaluado a través de la Escala Visual Analógica muestra cambios próximos a la significación, lo que sugiere que un aumento del tiempo de intervención podría poner de relieve resultados más relevantes. Tampoco se hallaron diferencias significativas en inteligencia emocional o autoconcepto, sin referentes previos que midan estos parámetros en pacientes con FM.

La ausencia de cambios significativos en la composición corporal, podría estar causada por la duración del estudio. Programas de actividad física aplicados a población con FM y otros específicos de danza con población sin FM que registran cambios en estos parámetros, tienen duraciones superiores al presente trabajo (Pantelic et al., 2013; Santos, 2013). Asimismo, la leve intensidad de trabajo podría estar influyendo en la evolución de este parámetro. Sería interesante poder ajustar en futuras líneas de trabajo la intensidad con la que se desarrollan estos ejercicios, para observar si modificaciones en este parámetro podría tener un mayor impacto la composición corporal y en otras medidas evaluadas.

Una de las principales limitaciones del trabajo expuesto es relativa a su duración, lo que pone de relieve la necesidad de contrastar los datos obtenidos futuras intervenciones más prolongadas. Otra limitación reside en que no existen referentes previos en la bibliografía que utilicen la danza española en pacientes con fibromialgia, lo cual limita la capacidad para discutir los resultados y hay que ser cautos a la hora de interpretarlos. Asimismo, en futuros trabajos se debería efectuar un análisis más exhaustivo de la condición física para obtener una perspectiva más completa de los cambios producidos por la actividad física en la muestra de estudio. Entre otras razones, por la relación existente entre la mejora de la condición física y factores como la depresión en personas sanas (Soriano-Maldonado, 2015), y que podrían ayudar a 


\section{Chica, A., González-Guirval, F., Reigal, R.E., Carranque, G. y Hernández-Mendo, A.}

interpretar los resultados obtenidos en pacientes con fibromialgia.

\section{APLICACIONES PRÁCTICAS}

Se sugiere que la aplicación de un programa normalizado de danza española en pacientes con fibromialgia podría incidir positivamente sobre aspectos como el estado de ánimo, la depresión y la ansiedad. Además, las mejoras en ansiedad y depresión podrían indicar que los patrones psicológicos son resultado y no causa de la fibromialgia (Arcos-Carmona et al., 2011), al disminuir tras aplicar el programa, a diferencia de otros estudios que hipotetízan que los síntomas ansioso-depresivos serían la causa de la enfermedad (Cuevas-Toro, López-Torrecillas, García-Medina y Díaz-Batanero, 2008).

\section{REFERENCIAS (APA 6 a EDICION)}

1. Altan, L., Korkmaz, N., Bingol, Ü., y Gunay, B. (2009). Effect of pilates training on people with fibromyalgia syndrome: a pilot study. Archives of physical medicine and Rehabilitation, 90(12), 1983-1988. doi:10.1016/j.apmr.2009.06.021

2. Aparicio, V. A., Segura-Jimenez, V., AlvarezGallardo, I. C., Soriano-Maldonado, A., CastroPinero, J., Delgado-Fernandez, M., y CarbonellBaeza, A. (2015). Fitness testing in the fibromyalgia diagnosis: the al-Andalus project. Medicine and Science in Sports and Exercise, 47(3), 451-459.

doi:10.1249/MSS.0000000000000445

3. Alpert, P. T. (2011). The health benefits of dance. Home Health Care Management \& Practice, 23(2), 155-157. doi:10.1177/1084822310384689

4. Arce, C., Andrade, E. M., y Seoane, G. (2000). Problemas semánticos en la adaptación del POMS al castellano. Psicothema, 12(2), 47-51.

5. Arcos-Carmona, I. M., Castro-Sánchez, A. M., Matarán-Peñarrocha, G. A., Gutiérrez-Rubio, A. B., Ramos-González, E., y Moreno-Lorenzo, C. (2011). Efectos de un programa de ejercicios aeróbicos y técnicas de relajación sobre el estado de ansiedad, calidad del sueño, depresión y calidad de vida en pacientes con fibromialgia: ensayo clínico aleatorizado. Medicina Clínica, 137(9), 398-401.

doi:10.1016/j.medcli.2010.09.045

6. Assis, M. R., Silva, L. E., Alves, A. M. B., Pessanha, A. P., Valim, V., Feldman, D., ... y Natour, J. (2006). A randomized controlled trial of deep water running: clinical effectiveness of aquatic exercise to treat fibromyalgia. Arthritis Care \& Research, 55(1), 57-65. doi:10.1002/art.21693

7. Beck, A.T., Ward, C.H.., Mendelson, M., Mock, H., y Erbaugh, J. (1961). An inventary for measuring depressión. Archives of General Psychiatry, 4, 561-571. doi:10.1001/archpsyc.1961.01710120031004

8. Bernardes, M.G. (2011). Influencia del baile recreativo en el estado de ánimo y autoestima de personas adultas. Eureka, 8(2), 241-253.

9. Blázquez, J. (2015). La actividad física con caballos como medio terapéutico para mejorar la capacidad funcional y la calidad de vida de las personas de la Tercera Edad y de las afectadas por el síndrome de Fibromialgia: El Método Centauro como programa formativo de intervención. Tesis doctoral. Universidad autónoma de Barcelona.

10. Busch, A. J., Schachter, C. L., Overend, T. J., Peloso, P. M., y Barber, K. A. (2008). Exercise for fibromyalgia: a systematic review. The Journal of Rheumatology, 35(6), 1130-1144.

11. Busse, J. W., Ebrahim, S., Connell, G., Coomes, E. A., Bruno, P., Malik, K., ... y Riva, J. J. (2013). Systematic review and network meta-analysis of interventions for fibromyalgia: a protocol. Systematic Reviews, 2(1), 18. doi:10.1186/20464053-2-18

12. Burckhardt, C., Clark, S., y Bennet, R. M. (1991). The fibromyalgia impact questionnaire: development and validation. The Journal of Rheumatology, 18(5), 728-733.

13. Carballo, M., Berral, F.J., Brandi, J.M., Campos, S., Cejudo, M. P., Galiano, D., ... y Romero, M. P. (2005). Fibromialgia: Proceso asistencial integrado. Sevilla: Junta de Andalucía.

14. Carbonell, A. (2010). Ejercicio físico en personas con fibromialgia. Efectos sobre el grado de dolor, 


\section{Danza española en mujeres con fibromialgia}

capacidad funcional y aspectos psicosociales. Tesis Doctoral Europea. Granada: Editorial de la Universidad de Granada.

15. Carbonell-Baeza, A., Aparicio, V. A., ÁlvarezGallardo, I. C., y Delgado-Fernández, M. (2014). Programa de ejercicio físico en fibromialgia. Sevilla: Junta de Andalucía.

16. Cuevas-Toro, A. M., López-Torrecillas, F., García-Medina, A., y Díaz-Batanero, M. D. C. (2008). Personalidad y estrategias de afrontamiento en pacientes con fibromialgia. Psicología Conductual, 16(2), 289-306.

17. Escudero, M. J., García, N., Prieto, M.A., Pérez, O., March, J.C., y López, M. (2010). Fibromialgia: percepción de pacientes sobre su enfermedad y el sistema de salud. Estudio de investigación cualitativa. Reumatología Clínica, 6(1), 16-22. doi:10.1016/j.reuma.2009.04.008

18. Estévez-López, F., Gray, C. M., Segura-Jiménez, V., Soriano-Maldonado, A., Álvarez-Gallardo, I. C., Arrayás-Grajera, M. J., ... y Pulido-Martos, M. (2015). Independent and combined association of overall physical fitness and subjective well-being with fibromyalgia severity: the al-Ándalus project. Quality of Life Research, 24(8), 18651873. doi:10.1007/s11136-015-0917-7

19. Fernandez-Berrocal, P., Extremera, N., y Ramos, N. (2004). Validity and reliability of the Spanish modified version of the Trait Meta-Mood Scale. Psychological Reports, 94(3), 751-755. doi:10.2466/pr0.94.3.751-755

20. Frade, J. A. (2011). Efectos de un programa de ejercicio vibratorio corporal sobre el equilibrio estático y dinámico, la fuerza, el dolor y la calidad de vida en mujeres con el síndrome de fibromialgia. Tesis doctoral. Universidad de Extremadura.

21. García, F., y Musitu, G. (2001). Autoconcepto Forma 5. AF5. Manual. Madrid: TEA.

22. Gardner, J. A. (1991). Early referral and other factors affecting vocational rehabilitation outcome for the workers' compensation client. Rehabilitation Counseling Bulletin, 34(2), 197209.
23. George, J. D., Fisher, A. G., y Vehrs, P. R. (2007). Tests y pruebas físicas (4a ed.). Barcelona: Paidotribo.

24. Góes, S. M., Leite, N., Shay, B. L., Homann, D., Stefanello, J. M., y Rodacki, A. L. (2012). Functional capacity, muscle strength and falls in women with fibromyalgia. Clinical Biomechanics, 27(6), 578-583. doi:10.1016/j.clinbiomech.2011.12.009

25. Goldenberg, D. L. (1999). Fibromyalgia syndrome a decade later: what have we learned? Archives of Internal Medicine, 159(8), 777-785. doi:10.1001/archinte.159.8.777

26. Häkkinen, A., Häkkinen, K., Hannonen, P., y Alen, M. (2001). Strength training induced adaptations in neuromuscular function of premenopausal women with fibromyalgia: comparison with healthy women. Annals of the Rheumatic Diseases, 60(1), 21-26. doi:10.1136/ard.60.1.21

27. Hamilton, M. (1959). The assessment of anxiety states by rating. British Journal of Medical Psychology, 32(1), 50-55. doi:10.1111/j.20448341.1959.tb00467.x

28. Humphrey, L., Arbuckle, R., Mease, P., Williams, D. A., Samsoe, B. D., y Gilbert, C. (2010). Fatigue in fibromyalgia: a conceptual model informed by patient interviews. BMC Musculoskeletal Disorders, 11(1), 216. doi: 10.1186/1471-247411-216

29. Humphrey, L., Arbuckle, R., Mease, P., Williams, D. A., Samsoe, B. D., y Gilbert, C. (2010). Fatigue in fibromyalgia: a conceptual model informed by patient interviews. BMC Musculoskeletal Disorders, 11(1), 216. doi: 10.1186/1471-247411-216

30. Keogh, J. W., Kilding, A., Pidgeon, P., Ashley, L., y Gillis, D. (2009). Physical benefits of dancing for healthy older adults: a review. Journal of Aging and Physical Activity, 17(4), 479-500. doi:10.1123/japa.17.4.479

31. Kingsley, J. D., Panton, L. B., Toole, T., Sirithienthad, P., Mathis, R., y McMillan, V. (2005). The effects of a 12-week strength-training program on strength and functionality in women with fibromyalgia. Archives of Physical Medicine 


\section{Chica, A., González-Guirval, F., Reigal, R.E., Carranque, G. y Hernández-Mendo, A.}

and Rehabilitation, 86(9), 1713-1721. doi:10.1016/j.apmr.2005.04.014

32. Knestaut, M., Devine, M. A., y Verlezza, B. (2010). It gives me purpose: The use of dance with people experiencing homelessness. Therapeutic Recreation Journal, 44(4), 289-301.

33. Lázaro, C., Bosch, F., Torrubia, R., y Banos, J. E. (1994). The development of a Spanish questionnaire for assessing pain: Preliminary data concerning reliability and validity. European Journal of Psychological Assessment, 10(2), 14551 .

34. Lobo, A., Chamorro, L., Luque, A., Dal-Ré, R., Badia, X., y Baró, E. (2002). Validation of the Spanish versions of the Montgomery-Asberg depression and Hamilton anxiety rating scales. Medicina Clinica, 118(13), 493-499. doi:10.1016/S0025-7753(02)72429-9

35. López-Rodríguez, M. M., Castro-Sánchez, A. M., Fernández-Martínez, M., Matarán-Peñarrocha, G. A., y Rodríguez-Ferrer, M. E. (2012). Comparación entre biodanza en medio acuático y stretching en la mejora de la calidad de vida y dolor en los pacientes con fibromialgia. Atención Primaria, 44(11), 641-649. doi:10.1016/j.aprim.2012.03.002

36. Palstam, A., Gard, G., y Mannerkorpi, K. (2013). Factors promoting sustainable work in women with fibromyalgia. Disability and Rehabilitation, 35(19), 1622-1629. doi:10.3109/09638288.2012.748842

37. McNair, D. M., Lorr, M., y Droppleman, L. E (1971). Manual for the Profile of Mood States (POMS). San Diego, CA: Educational and Industrial Testing Service.

38. Monterde, S., Salvat, I., Montull, S., y Fernández, J. (2004). Validación de la versión

39. española del Fibromyalgia Impact Questionnaire. Revista Española de Reumatología, 31 (9), 507513.

40. Munguía, D., Alegre, C., Serverto, O., y Legaz, A. (2007). Eficacia de los tratamientos en el síndrome de fibromialgia. En, D, Munguía, A., Legaz y C., Alegre, (Eds), Guía práctica clínica sobre el síndrome de fibromialgia para profesionales de la salud. (pp. 11-24). Madrid: Elsevier.
41. Nørregaard, J., Lykkegaard, J. J., Mehlsen, J., y Danneskiold-Samsøe, B. (1997). Exercise training in treatment of fibromyalgia. Journal of Musculoskeletal Pain, 5(1), 71-79. doi:10.1300/J094v05n01_05

42. Pantelic, S., Milanovic, Z., Sporis, G., y Stojanovic-Tosic, J. (2013). Efectos de los Ejercicios de danza aeróbica durante doce semanas sobre los parámetros de composición corporal en mujeres jóvenes. International Journal of Morphology, 31(4), 1243-1250. doi:10.4067/S0717-95022013000400016

43. Peluso, M.A., y Andrade, L.H. (2005). Physical activity and mental health: the association between exercise and mood, Clinics, 60(1), 61-70. doi:10.1590/S1807-59322005000100012

44. Pinniger, R., Thorsteinsson, E. B., Brown, R. F., y McKinley, P. (2013). Tango dance can reduce distress and insomnia in people with self-referred affective symptoms. American Journal of Dance Therapy, 35(1), 60-77. doi:10.1007/s10465-0129141-y

45. Pinzón, I. D., Angarita, A., y Correa, E. A. (2013). Efectos de un programa de entrenamiento funcional en la musculatura core en mujeres con fibromialgia. Revista de Ciencias de la Salud, $13(1)$, 39-53. doi:10.12804/revsalud13.01.2015.03

46. Reca, M. (2005). El cuerpo en la danza: Que es Danza Terapia Movimiento. Buenos Aires: Lumen.

47. Restrepo, J.C., y Rojas, J.G. (2011). Fibromialgia.i Qué deben saber y evaluar los profesionales de Enfermería?. Investigación $y$ Educación en Enfermería, 29(2), 305-314.

48. Rodham, K., Rance, N., y Blake, D. (2010). A qualitative exploration of carers' and 'patients' experiences of fibromyalgia: one illness, different perspectives. Musculoskeletal Care, 8(2), 68-77. doi:10.1002/msc.167

49. Rodríguez, R.M., Orta, M.A., y Amashta, L. (2010). Cuidados de enfermería a los pacientes con fibromialgia. Enfermería Global, 9(2), 1-16. doi:10.4321/S1695-61412010000200010 


\section{Danza española en mujeres con fibromialgia}

50. Romero, A. (2012). Efectos de la práctica del Taichi en mujeres y hombres con fibromialgia. Tesis doctoral. Universidad de Granada.

51. Santos, M. A. (2013). Efectos de un programa combinado: seco y agua, sobre el impacto de la enfermedad, la composición corporal, la condición física y salud y calidad de vida de mujeres con fibromialgia. Tesis doctoral. Universidad de Jaén.

52. Sencan, S., Ak, S., Karan, A., Muslumanoglu, L., Ozcan, E., y Berker, E. (2004). A study to compare the therapeutic efficacy of aerobic exercise and paroxetine in fibromyalgia syndrome. Journal of Back and Musculoskeletal Rehabilitation, 17(2), 57-61. doi:10.3233/BMR2004-17204

53. Salovey, P., Mayer, J. D., Goldman, S., Turvey, C., y Palfai, T. (1995). Emotional attention, clarity, and repair: Exploring emotional intelligence using the Trait Meta-Mood Scale. En J. W. Pennebaker (Ed.), Emotion, disclosure, and health (pp. 125-154). Washington, DC: American Psychological Association. doi:10.1037/10182006

54. Shavelson, R., Hubner, J., y Stanton, J. (1976). Self concept: Validation of construct interpretation. Review of Educational Research, $46 \quad$ (3), 407-441. doi:10.3102/00346543046003407

55. Soriano-Maldonado, A. (2015). Percepción del esfuerzo, condición fisica, depresión y severidad de la enfermedad en mujeres con fibromialgia. Tesis Doctoral. Universidad de Granada.

56. Spielberger, C. D. (1983). Manual for the StateTrait Anxieg Inventori STAI (Form Y). Palo Alto, CA: Consulting Psychologists Press.

57. Tomas-Carus, P., Gusi, N., Häkkinen, A., Häkkinen, K., Leal, A., y Ortega-Alonso, A. (2008). Eight months of physical training in warm water improves physical and mental health in women with fibromyalgia: a randomized controlled trial. Journal of Rehabilitation Medicine, 40(4), 248-252. doi:10.2340/165019770168

58. World Health Organization. Classification of diseases (2010). Disponible en: http://apps.who.int/classifications/icd10/browse/2 010/en, [Consulta: 30 de mayo de 2015].

59. World Medical Association. Declaration of Helsinki (2013). Disponible en http://www.wma.net/en/20activities/10ethics/10h elsinki/, [Consulta: 23 de marzo de 2015]. 